\title{
Variability of relative cerebral blood volume measurements of recurrent glioma
}

\author{
Laura K. Reed ${ }^{1,2}$, Jason H. Huang ${ }^{1,2}$ \\ ${ }^{1}$ Department of Neurosurgery, Baylor Scott \& White Health, Scott and White Medical Center, Temple, TX, USA; ${ }^{2}$ Department of Surgery, Texas \\ A\&M University College of Medicine, Temple, TX, USA \\ Correspondence to: Jason H. Huang, MD, FACS. Department of Neurosurgery, Baylor Scott \& White Health, Scott and White Medical Center, 2401 S. \\ 31st Street, Temple, TX 76508, USA. Email: jason.huang@bswhealth.org. \\ Provenance: This is an invited article commissioned by the Editorial Office, Annals of Translational Medicine. \\ Comment on: Smits M, Bendszus M, Collette S, et al. Repeatability and reproducibility of relative cerebral blood volume measurement of recurrent \\ glioma in a multicentre trial setting. Eur J Cancer 2019;114:89-96.
}

Submitted Nov 25, 2019. Accepted for publication Dec 05, 2019.

doi: $10.21037 /$ atm.2019.12.41

View this article at: http://dx.doi.org/10.21037/atm.2019.12.41

Primary central nervous system (CNS) tumors are a leading cause of death worldwide, with an incidence of 7.08 per 100,000 and an average of 15,944 deaths per year in the United States alone. The 5 -year survival rate is $35.8 \%$ for all malignant CNS tumors, and only 6.8\% for glioblastomas (1); thus, determining a method of monitoring effectiveness of treatment and recurrence is of utmost importance. For example, patients with glioblastoma undergo neurosurgical maximal safe resection followed by radiotherapy and chemotherapy with temozolomide, a DNA alkylating agent. A sequela of the radiotherapy is pseudoprogression, or radiation necrosis, that appears as increased tumor size and mass effect on post-treatment MRIs. Pseudoprogression is difficult to distinguish from tumor recurrence, complicating the decision to continue the adjuvant chemotherapy temozolomide or to add bevacizumab which targets angiogenesis. Dynamic susceptibility contrast (DSC) MRI can be used to measure the relative cerebral blood volume $(\mathrm{rCBV})$ and thus differentiate between necrosis (decreased $\mathrm{rCBV}$ ) and recurrence (increased) to guide treatment or assist with prognostication (2).

Smits et al. aimed to determine whether there was enough consistency between three neuroradiologists (intra- and inter-observer) at three different medical centers to define a rCBV threshold for radiation necrosis versus recurrence was reproducible and could have clinical significance. The radiologists reviewed images from 17 patients twice over the course of 1 year and found the area of maximum $\mathrm{rCBV}$ in the region of interest (ROI) using normal appearing white matter in the centrum semiovale as the reference tissue. Two of the three radiologists consistently measured similar rCBV values on repeat review of each image and one had considerable variability. One reviewer did not acquire the maximum $\mathrm{rCBV}$ but reported the average in the ROI. Ultimately, Smits determined that there is not sufficient reproducibility and repeatability in measuring $\mathrm{rCBV}$ to guide clinical decisions.

DSC MRI is a T2-based first pass image of gadolinium contrast through vasculature which causes a signal drop due to susceptibility effects of the bolus and thus yields an image reflecting perfusion (3). In order to quantify perfusion accurately, the blood brain barrier must be intact. Thus, after treatment, the area of interest can appear larger since the barrier has been disrupted (4) making it difficult to determine whether that area is radiation necrosis or recurrence. The $\mathrm{rCBV}$ is acquired from DSC MRI as true CBV cannot be directly measured. CBV varies with cardiac output, hematocrit, acquisition protocols and postprocessing software and must be normalized to reference tissue (5). Selection of reference tissue has varied in prior studies and it was determined that the contralateral normal appearing white matter in the centrum semiovale yields the least variability (5) which is the reference tissue chosen by Smits et al. Theoretically, the rCBV should only be higher in areas of recurrence due to the increased angiogenesis and areas of radionecrosis should be comparable to normal 
tissue $(4,6)$. DSC MRI is not accurate when near bone or large vessels, which was taken into account when choosing the gliomas to analyze in this study. The other challenge to consider is the heterogeneity of the masses which could allow error to be introduced when multiple observers are selecting regions of interest.

Dijkstra et al. proved that out of all the parameters to compare on MRI, rCBV was the most reproducible between observers (other parameters included apparent diffusion coefficient, maximum contrast enhancement, time to peak, wash in rate, and wash out rate) (7). They also determined that single slice ROI selection is not significantly different from attempting to capture the whole tumor in a $3 \mathrm{D}$; thus, $2 \mathrm{D}$ selection is sufficient. However, in this study, the ROI was selected freeform around the largest area of the tumor instead of using the area of maximum rCBV as in Smits' study; it cannot be determined whether one of these methods is superior. In another study that tried to determine a threshold between low- and high-grade gliomas, the heterogeneity of the tumors was thought to be the largest obstacle when measuring maximum rCBV (8), which could support a freeform method over selecting the best part of the tumor to fit inside a set ROI. Dijkstra et al. largely supports the techniques used in Smits' and showed that complex measurements in $3 \mathrm{D}$ are not required, single slice measurements are equally as valuable. One must also consider the significant variability in the acquisition of the imaging such as magnetic field strength, leakage correction methods, and post-processing that must be considered when attempting to standardize measurements that dictate oncological treatments.

In order to use this methodology for guiding treatment, one must consider the timing of the image acquisition as most high-grade gliomas have an average recurrence time of 6.9 months (6). As expected, those with a negative DSC, or one with a rCBV comparative to the normal white matter, have a longer survival. Multiple studies have tried to apply a numerical value to the transition between lowor high-grade glioma (8) or between radiation necrosis and recurrence (4), but there has not been a consensus in the appropriate values to assign to each category.

Overall, Smits et al. designed an appropriate study to further investigate the reproducibility and repeatability of rCBV measurements. They were the first to study rCBV across multiple institutions and multiple radiologists. However, expanding the breadth revealed multiple problems with the measurement of rCBV. Firstly, the radiologists were not consistent with their measurements when they analyzed the images $2-11$ months apart which proves the subjective nature of determining an accurate and precise rCBV. One reviewer did not measure the same rCBV on repeat review of the images and another reported an average ROI instead of the maximum. Another shortcoming of this study was the limited sample size as only 12 patients had ideal imaging and another 5 were used despite the presence of hemorrhage and white matter lesions in the contralateral tissue. As discussed by Kwee et al. blood products can significantly affect the assessment due to the increased susceptibility artifacts (4). Provider adherence to the study protocol and improved patient selection would have increased the validity of the study. Despite these discrepancies, at least 2 of the 3 reviewers did have measurements of $\mathrm{rCBV}$ that were in the same category for each tumor (reduced, minimal, moderate, strong increase). To apply this concept clinically, each set of images would need to be reviewed by multiple radiologists before a treatment decision could be made.

Smits et al. ultimately exposed the difficulty in applying a single measurement to a heterogeneous patient population by various radiologists and proved the need for further research with strict regulations. The lack of adherence to the protocol by the reviewers, differences in repeat measurements, and inconsistent patient selection allow one to question the validity of this study. If another were to be performed, increasing the sample size, standardizing the MRI acquisition, post-processing, and analysis by the radiologists would be imperative. There is too much variation across all studies to determine a clinically useful rCBV measurement to guide the treatment plans of patients with gliomas. Perhaps the DSC MRI imaging could be used to trend post-treatment rCBV changes and assist with clinical decision making if there appears to be a stable or an upward trend designating recurrence.

One option would be to use additional imaging modalities to determine the significance of the findings in DSC MRI. T1-weighted contrast enhanced MRI is only useful 24-72 hours after surgery as the enhancement can be caused by inflammation or ischemia later during treatment; therefore, contrast MRI cannot be reliably used to assess recurrence versus radiation necrosis (9). MR spectroscopy and perfusion MRI have been shown to be the most sensitive and specific for delineating between the possible diagnoses (10). Even higher accuracy can be obtained with positron emission tomography (PET) and the tracer C-methyl-L-methionine (C-MET) however due to the short half-life the cyclotron must be at the same site 
the tracer is injected. O-(2-(18)F-fluoroethyl)-L-tyrosine (F-FET) is another tracer which was proven to be equally as accurate as $\mathrm{C}-\mathrm{MET}$ in predicting radiation necrosis and the longer half-life allows for wider application and the ability to travel for image acquisition; therefore, F-FET is the best tracer for this application. The most common tracer, fluorodeoxyglucose (FDG), is not preferred in cerebral imaging as it is difficult to distinguish between normal brain with high glucose uptake and tumor tissue with increased uptake (9). In order to verify the accuracy of imaging modalities, a study would need to be performed correlating the imaging findings to the histopathology for confirmation. As of now, there is not a simple way to delineate between radiation necrosis and recurrence, clinicians must continue to rely on all available resources and their best judgment when designing treatment plans for these patients.

\section{Acknowledgments}

Funding: This work was supported, in part, by NIH-R01NS-067435 (JH Huang) and Helen Vosburg McCrillus Plummer and Robert Edward Lee Plummer, Jr. Chair in Neurosurgery (JH Huang).

\section{Footnote}

Conflicts of Interest: The authors have no conflicts of interest to declare.

Ethical Statement: The authors are accountable for all aspects of the work in ensuring that questions related to the accuracy or integrity of any part of the work are appropriately investigated and resolved.

\section{References}

1. Ostrom QT, Cioffi G, Gittleman H, et al. CBTRUS statistical report: primary brain and other central nervous system tumors diagnosed in the United States in 2012-

Cite this article as: Reed LK, Huang JH. Variability of relative cerebral blood volume measurements of recurrent glioma. Ann Transl Med 2019;7(Suppl 8):S260. doi: 10.21037/ atm.2019.12.41
2016. Neuro Oncol 2019;21:v1-100.

2. Omuro A, DeAngelis LM. Glioblastoma and other malignant gliomas: a clinical review. JAMA 2013;310:1842-50.

3. Saini J, Gupta RK, Kumar M, et al. Comparative evaluation of cerebral gliomas using rCBV measurements during sequential acquisition of T1-perfusion and T2*perfusion MRI. PLoS One 2019;14:e0215400.

4. Kwee RM, Kwee TC. Dynamic susceptibility MR perfusion in diagnosing recurrent brain metastases after radiotherapy: a systematic review and meta-analysis. J Magn Reson Imaging 2019. [Epub ahead of print].

5. Oei MTH, Meijer FJA, Mordang JJ, et al. Observer variability of reference tissue selection for relativecerebral blood volume measurements in glioma patients. Eur Radiol 2018;28:3902-11.

6. Fong C, Parpia S, Yemen B, et al. Using magnetic resonance perfusion to stratify overall survival in treated high-grade gliomas. Can J Neurol Sci 2019;46:533-9.

7. Dijkstra H, Sijens PE, van der Hoorn A, et al. Interobserver reproducibility of quantitative dynamic susceptibility contrast and diffusion MRI parameters in histogram analysis of gliomas. Acta Radiol 2019. [Epub ahead of print].

8. Soliman RK, Gamal SA, Essa AA, et al. Preoperative grading of glioma using dynamic susceptibility contrast MRI: relative cerebral blood volume analysis of intratumoural and peri-tumoural tissue. Clin Neurol Neurosurg 2018;167:86-92.

9. Zikou A, Sioka C, Alexiou GA, et al. Radiation necrosis, pseudoprogression, pseudoresponse, and tumor recurrence: imaging challenges for the evaluation of treated gliomas. Contrast Media Mol Imaging 2018;2018:6828396.

10. van Dijken BRJ, van Laar PJ, Holtman GA, et al. Diagnostic accuracy of magnetic resonance imaging techniques for treatment response evaluation in patients with high-grade glioma, a systematic review and metaanalysis. Eur Radiol 2017;27:4129-44. 